\title{
A coming of age for immune checkpoint inhibitors in cancer
}

\author{
Ryan D Nipp ${ }^{1}$ \& Justin F Gainor*,1 \\ ${ }^{1}$ Department of Medicine, Division of Hematology \& Oncology, Massachusetts General Hospital Cancer Center \& Harvard Medical \\ School, Boston, MA, USA \\ *Author for correspondence: Tel.: +1 617724 4000; Fax: +1 617643 0458; jgainor@partners.org
}

' ${ }^{i m m}$ munotherapy has a wide range of possible serious adverse effects, but also the potential for long-lasting benefits, yet both the risks and benefits are incompletely understood for the geriatric oncology population."

First draft submitted: 26 April 2019; Accepted for publication: 29 April 2019; Published online: 15 May 2019

Keywords: age $\bullet$ chemotherapy $\bullet$ immune checkpoint inhibitors $\bullet$ immunotherapy $\bullet$ melanoma

\section{Cancer \& aging}

Older adults represent a rapidly growing population in oncology, and they frequently present with complex concurrent medical issues [1]. Oncologists often encounter challenges when caring for older adults with cancer due to their multifaceted constellation of medical and psychosocial issues. Notably, studies suggest that older patients with cancer experience worse survival outcomes compared with younger patients, possibly due to suboptimal treatment $[2,3]$. Importantly, although data support that older adults derive similar benefits from chemotherapy compared with younger patients, older patients also have greater risk for experiencing side effects from chemotherapy, which may diminish oncologists' enthusiasm for offering such treatment to the geriatric oncology population $[4,5]$. Indeed, oncologists often seek more tolerable treatment options that can benefit older patients while avoiding unwanted side effects [6,7]. Older patients' increased toxicity from chemotherapy may be related to concomitant comorbid conditions, greater risk of polypharmacy, diminished functional reserves and lack of social support [5]. However, not all older adults have the same risk of experiencing poor outcomes and some may do well with treatment, thus reflecting a population with unique and varied needs $[6,8]$.

\section{Cancer \& immune checkpoint inhibitors}

In recent years, immunotherapies directed against negative regulators of the immune response have revolutionized the treatment paradigm for many patients with cancer. In particular, monoclonal antibodies targeting the programmed cell death 1 receptor (PD-1) and its ligand, PD-L1, have exhibited impressive activity across an evergrowing list of malignancies. Indeed, within a matter of just 5 years, $\mathrm{PD}-(\mathrm{L}) 1$ inhibitors have become standard therapies in $>10$ indications, ranging from melanoma to non-small-cell lung cancer to lymphoma. Currently, six PD-(L) 1 inhibitors have gained regulatory approval in the USA, and there are ongoing efforts to expand the use of these agents to other malignancies and new disease settings (e.g., adjuvant therapy). In parallel, attempts to develop more effective immune-based combinations have become a major research focus throughout oncology.

Enthusiasm for the use of PD-(L) 1 inhibitors stems from robust data demonstrating that these agents can induce significant, and sometimes durable, anti-tumor immune responses [9]. In general, PD-(L)1 inhibitors also have a more favorable side-effect profile compared with traditional cytotoxic chemotherapy [10]. Nonetheless, immune checkpoint inhibitors may also give rise to a unique spectrum of toxicities, known as immune-related adverse events (irAEs). While most irAEs are relatively mild to moderate, and transient in nature when promptly recognized, severe and/or even fatal toxicities have been observed. This has prompted ongoing efforts to develop consensus guidelines for the management of irAEs. Collectively, these trends underscore that the use of immunotherapy in everyday clinical practice will continue to expand and oncologists will need to be prepared for their growing use.

Future Medicine 


\section{Aging \& immune checkpoint inhibitors}

Older adults represent the largest group of patients with cancer; thus, it is critical to understand the risks and benefits of novel cancer treatment in the geriatric oncology population [1,11]. Historically, older adults are underrepresented in cancer clinical trials despite their representation as the most commonly encountered patient in routine clinical practice [12]. Reasons for the underrepresentation of older adults in cancer clinical trials are myriad and include issues related to trial availability, rigid inclusion/exclusion criteria and even oncologists' hesitancy to discuss clinical trials with older patients. For these reasons, the evidence for how best to care for the rapidly growing geriatric oncology population is limited. Specifically, the data for using novel immunotherapy agents to treat older patients with cancer are sparse and limited largely to meta-analyses and retrospective studies [13-16].

Most of the available evidence for treating older patients with immune checkpoint inhibitors suggests that older adults with cancer experience clinical benefits. A meta-analysis investigating the benefits of checkpoint inhibitors in older adults with cancer age 65 and above identified 53 articles, 17 of which employed a randomized controlled study design [15]. Out of the studies included in this meta-analysis, various cancer types were represented, and multiple immune checkpoint inhibitors were used (e.g., nivolumab, pembrolizumab and atezolizumab). Importantly, improvements in overall survival were consistent for patients older and younger than 65 years who were treated with immune checkpoint inhibitors. Recently, Nishijima and colleagues performed a similar meta-analysis of nine randomized controlled trials, again with varying cancer types, to examine the impact of age on outcomes with CTLA4 (ipilimumab and tremelimumab) and PD1 inhibitors (nivolumab and pembrolizumab) [16]. This study also demonstrated that immunotherapy resulted in significantly improved overall survival in both younger and older patients. An important limitation of both analyses is the lack of details on tolerance and toxicity of immunotherapy in older adults.

To complement the above meta-analyses, several recent retrospective studies have been performed among older patients treated in real-world practice. One study focused on patients with melanoma and investigated the impact of age on survival outcomes and rates of immune-mediated toxicities in patients treated with anti-PD-1/anti-PD-L1 monoclonal antibodies at two academic medical centers [13]. Although this was a retrospective study, this work included broad age ranges to help clinicians understand differences across subgroups of ages: $<50,50-64,65-74$ and $\geq 75$ years. Importantly, this study demonstrated that immunotherapy has the potential to provide beneficial effects in patients with advanced melanoma independent of age. Moreover, the investigators also studied adverse effects from immunotherapy and did not find significant differences in toxicity between older and younger patients, although arthritis was more common in older patients. Another retrospective study of real-world patients with lung cancer investigated immune checkpoint inhibitor benefits and side effects across ages $<60,60-69,70-79$ and $\geq 80$ years [14]. Interestingly, this study found that the oldest patients (age $\geq 80$ ) experienced worse survival outcomes than the younger age groups, yet irAEs, steroid use and hospitalizations did not differ by age. Thus, much of the available evidence suggests that PD-(L) 1 inhibitors are well tolerated among older adults with cancer, but additional work is needed to definitively determine the benefits of these agents in the geriatric oncology population.

\section{Future perspective}

The evidence supporting the use of immunotherapy continues to grow, and so too does the number of older adults who are diagnosed with cancer. As a result, numerous important issues remain to be studied, with some outlined here:

\section{Need for prospective studies focused on older adults with cancer}

Much of the current work describing the use of immunotherapy in the geriatric oncology population involves secondary data analyses and retrospective studies. As a result, we lack information about the benefits and risks of immunotherapy for the average older patient seen in routine practice. For example, secondary analyses of randomized trials often lack patients over age 75 , and these studies frequently exclude patients with certain comorbid conditions and/or functional impairment. Conversely, although retrospective studies of real-world patients can investigate patients at older ages and those with varying degrees of comorbidity/function, these studies lack a control arm of older patients who did not receive immunotherapy. Thus, these retrospective studies often compare outcomes between older versus younger patients, but we lack data for the counterfactual older adult who did not receive immunotherapy. Ultimately, prospective clinical trials are needed to investigate the impact of immunotherapy specifically focused on the geriatric oncology population. 


\section{Need to investigate potential predictors of benefits \&/or toxicities associated with immune checkpoint inhibitors}

A critical need exists (for both the geriatric oncology population as well as the general cancer population) to identify factors that can predict both response to treatment and potential adverse effects from immunotherapy. For older adults with cancer, potential predictors include geriatric-specific factors (e.g., comorbidity and functional independence), tumor-specific factors (e.g., tumor mutation burden [TMB]), and biomarkers of aging/immune dysregulation. Older adults are not a homogenous population, and thus it is imperative to assess these patients' comorbidity, functional independence, nutrition and psychosocial supports [17]. Importantly, geriatricians have developed tools to help assess and manage older patients' distinct geriatric concerns, but we lack data about whether these tools can predict response/toxicity associated with immunotherapy. Moreover, as patients age so too does the potential for immune dysregulation that may in term limit the effectiveness of immune checkpoint inhibitors [18]. Importantly, this may be counterbalanced by age-related increases in TMB within some tumor types [19]. TMB has been demonstrated to correlate with activity of immune checkpoint inhibitors across a range of malignancies [20]. Ultimately, additional work is urgently needed to identify factors to predict benefits and/or toxicities associated with immunotherapy, and a particular focus on geriatric-specific issues will be important when evaluating potential predictive factors among older adults with cancer.

\section{Need to study ways to enhance shared decision-making approaches in the geriatric oncology population related to novel cancer treatment}

Oncologists often struggle to accurately predict the risks and benefits of novel treatment options. However, a key role of the clinician involves preparing patients and their loved ones for the multitude of different possible outcomes. Notably, patients frequently report overly optimistic perceptions of the potential benefits of treatment, which can complicate their ability to make informed treatment decisions [21]. Additionally, patient-reported outcomes, such as quality of life, symptom burden and illness understanding are largely understudied among patients receiving immune checkpoint inhibitors, and these represent key components of shared decision-making. Moreover, immunotherapy has a wide range of possible serious adverse effects, but also the potential for long-lasting benefits, yet both the risks and benefits are incompletely understood for the geriatric oncology population. Thus, with immune checkpoint inhibitors and their unique toxicity profiles, additional research is needed to understand how best to communicate the possible range of outcomes with patients and their caregivers to enhance shared decision-making.

\section{Conclusion}

The geriatric oncology population represents a growing and diverse group of patients that is understudied and often excluded from cancer clinical trials. As the use of immune checkpoint inhibitors continues to increase, and these therapies revolutionize the treatment landscape in oncology, the need for rigorous, high-quality research to understand the risks and benefits of immunotherapies for older adults with cancer will continue to grow. Future efforts to enhance the care delivered to older adults with cancer will require focused attention to the unique needs of the geriatric oncology population and continued investment in research targeting this rapidly growing and often complex population of patients.

\section{Financial \& competing interests disclosure}

JF Gainor has served as a compensated consultant or received honoraria from Bristol-Myers Squibb, Genentech/Roche, Ariad/Takeda, Loxo, Pfizer, Incyte, Novartis, Merck, Agios, Amgen, Regeneron, Oncorus, Array, Jounce and Clovis Oncology. RD Nipp has no relevant financial relationships to declare. The authors have no other relevant affiliations or financial involvement with any organization or entity with a financial interest in or financial conflict with the subject matter or materials discussed in the manuscript apart from those disclosed.

No writing assistance was utilized in the production of this manuscript.

\section{References}

1. Yancik R. Population aging and cancer: a cross-national concern. Cancer J. 11(6), 437-441 (2005).

2. Patel SS, Nelson R, Sanchez J et al. Elderly patients with colon cancer have unique tumor characteristics and poor survival. Cancer 119(4), 739-747 (2013).

3. Bouchardy C, Rapiti E, Fioretta G et al. Undertreatment strongly decreases prognosis of breast cancer in elderly women. J. Clin. Oncol. 21(19), 3580-3587 (2003). 
4. Muss HB, Berry DA, Cirrincione C et al. Toxicity of older and younger patients treated with adjuvant chemotherapy for node-positive breast cancer: the Cancer and Leukemia Group B Experience. J. Clin. Oncol. 25(24), 3699-3704 (2007).

5. Hurria A, Wong FL, Villaluna D et al. Role of age and health in treatment recommendations for older adults with breast cancer: the perspective of oncologists and primary care providers. J. Clin. Oncol. 26(33), 5386-5392 (2008).

6. Extermann M, Hurria A. Comprehensive geriatric assessment for older patients with cancer. J. Clin. Oncol. 25(14), 1824-1831 (2007).

7. Azzoli CG, Temin S, Aliff T et al. 2011 Focused Update of 2009 American Society of Clinical Oncology clinical practice guideline update on chemotherapy for stage IV non-small-cell lung cancer. J. Clin. Oncol. 29(28), 3825-3831 (2011).

8. Corre R, Greillier L, Le Caer $\mathrm{H}$ et al. Use of a comprehensive geriatric assessment for the management of elderly patients with advanced non-small-cell lung cancer: the Phase III randomized ESOGIA-GFPC-GECP 08-02 study. J. Clin. Oncol. 34(13), 1476-1483 (2016).

9. Gong J, Chehrazi-Raffle A, Reddi S, Salgia R. Development of PD-1 and PD-L1 inhibitors as a form of cancer immunotherapy: a comprehensive review of registration trials and future considerations. J. Immunother. Cancer 6(1), 8 (2018).

10. Nishijima TF, Shachar SS, Nyrop KA, Muss HB. Safety and tolerability of PD-1/PD-L1 inhibitors compared with chemotherapy in patients with advanced cancer: a meta-analysis. Oncologist 22(4), 470-479 (2017).

11. Shapiro CL. Cancer survivorship. N. Eng. J. Med. 379(25), 2438-2450 (2018).

12. Townsley CA, Selby R, Siu LL. Systematic review of barriers to the recruitment of older patients with cancer onto clinical trials. J. Clin. Oncol. 23(13), 3112-3124 (2005).

13. Betof AS, Nipp RD, Giobbie-Hurder A et al. Impact of age on outcomes with immunotherapy for patients with melanoma. Oncologist 22(8), 963-971 (2017).

14. Lichtenstein MRL, Nipp RD, Muzikansky A et al. Impact of age on outcomes with immunotherapy in patients with non-small cell lung cancer. J. Thoracic Oncol. 14(3), 547-552 (2019).

15. Elias R, Giobbie-Hurder A, Mccleary NJ, Ott P, Hodi FS, Rahma O. Efficacy of PD-1 \& PD-L1 inhibitors in older adults: a meta-analysis. J. Immunother. Cancer 6(1), 26 (2018).

16. Nishijima TF, Muss HB, Shachar SS, Moschos SJ. Comparison of efficacy of immune checkpoint inhibitors (ICIs) between younger and older patients: a systematic review and meta-analysis. Cancer Treatment Rev. 45, 30-37 (2016).

17. Nipp RD, El-Jawahri A, Traeger $L$ et al. Differential effects of early palliative care based on the age and sex of patients with advanced cancer from a randomized controlled trial. Palliative Med. doi:10.1177/0269216317751893 269216317751893 (2018) (Epub ahead of print).

18. Naylor K, Li G, Vallejo AN et al. The influence of age on T cell generation and TCR diversity. J. Immunol. 174(11), 7446-7452 (2005).

19. Chalmers ZR, Connelly CF, Fabrizio D et al. Analysis of 100,000 human cancer genomes reveals the landscape of tumor mutational burden. Genome Med. 9(1), 34 (2017).

20. Yarchoan M, Hopkins A, Jaffee EM. Tumor mutational burden and response rate to PD-1 inhibition. N. Eng. J. Med. 377(25), 2500-2501 (2017).

21. Nipp RD, Greer JA, El-Jawahri A et al. Coping and prognostic awareness in patients with advanced cancer. J. Clin. Oncol. doi:10.1200/JCO.2016.71.3404 JCO2016713404 (2017) (Epub ahead of print). 